\title{
MAGNETIC POLARON IN A FERROMAGNETIC SEMICONDUCTOR. A NEW SELF-CONSISTENT FINITE TEMPERATURE SOLUTION
}

\author{
D.I. MARVAKOV ${ }^{1}$, A.L. KUZEMSKY \\ Joint Institute for Nuclear Research, Dubna, USSR \\ and \\ J.P. VLAHOV \\ Faculty of Physics, University of Sofia, Sofia, Bulgaria
}

Received 10 August 1984

A complete self-consistent finite temperature theory of magnetic polaron states with damping in a ferromagnetic semiconductor is developed using the irreducible Green function (IGF) method.

The $s-f$ hamiltonian is the simplest theoretical model to study magnetically ordered semiconductors. Recently a great deal of effort has been made to understand the relationship and mutual influence of both magnetic and band electron subsystems [1]. A very interesting problem is the forming of bound polaronlike states due to the effective attraction of the electron and magnon. This is possible for the case of antiferromagnetic coupling of the electron spin to the lattice (magnetic subsystem).

The properties of the magnetic polaron states have been investigated at zero temperature in refs. [2-4]. Recently a much more detailed theory of the magnetic polaron at $\boldsymbol{T}=0$ has been given in ref. [5] and the states of the current carriers in ferromagnetic semiconductors have been considered for arbitrary values of the $s-f$ exchange parameter $I$ in the spin wave region by a variational procedure in ref. [6]

The purpose of our paper is to develop a unified and complete self-consistent finite temperature theory of the magnetic polaron by taking explicitly into account the damping effects and finite lifetime. For this aim we use the novel irreducible Green function method proposed earlier in refs. $[7,8]$. The IGF method com-

1 Permanent address: Faculty of Physics, University of Sofia, Sofia, Bulgaria. pletely describes the quasiparticle inelastic scattering processes in many-body systems and gives the quasiparticle spectra with damping in a very general way.

The one-electron GF [9] $G_{k \sigma}(\omega)=\left\langle\left\langle a_{k \sigma} \mid a_{k \sigma}^{+}\right\rangle\right\rangle$ can be calculated in the low-concentration limit in the following form

$G_{k \sigma}(\omega)=\left\{\left[G_{k \sigma}^{0}(\omega)\right]^{-1}-\Sigma_{\sigma}(k, \omega)\right\}^{-1}$

where the generalized mean-field GF $G_{k \sigma}^{0}(\omega)$ is

$G_{k \sigma}^{0}(\omega)=\left[\omega-\epsilon_{k \sigma}-\left(I^{2} / N\right) \psi_{k \sigma}(\omega)\right]^{-1}$.

Here $\psi_{k \sigma}(\omega)$ means

$$
\begin{gathered}
\psi_{k \sigma}(\omega)=\sum_{a}\left(\frac{\left\langle S_{-q}^{-\sigma} S_{q}^{\sigma}\right\rangle}{\left[1-I \Lambda_{k \sigma}(\omega)\right]\left[\omega+Z_{\sigma} \omega_{q}-\epsilon_{k+q,-\sigma}\right]}\right. \\
\left.+\frac{\left[1+I \Lambda_{k \sigma}(\omega)\right]\left\langle\left(S_{-q}^{Z}\right)^{\mathrm{ir}}\left(S_{q}^{Z}\right)^{\mathrm{ir}}\right\rangle}{\left[1-I \Lambda_{k \sigma}(\omega)\right]\left[\omega-\epsilon_{k+q, \sigma}\right]}\right)
\end{gathered}
$$

where

$\Lambda_{k \sigma}(\omega)=N^{-1} \sum_{q}\left(\omega+Z_{\sigma} \omega_{q}-\epsilon_{k+q,-\sigma}\right)^{-1}$,

$\epsilon_{\boldsymbol{k} \sigma}$ is the single-particle electron energy, $\omega_{\boldsymbol{q}}$ denotes the magnon energy and $Z_{\sigma}=1(-1)$ if $\sigma=\uparrow(\downarrow)$ or $+(-)$. Our generalized mf GF $G_{k \sigma}^{0}(\omega)$ is exactly reduced to the Shastry-Mattis [5] solution at $T=0$ : 
$G_{0}^{\mathrm{SM}}=\left(\omega-\epsilon_{k \sigma}-\frac{\delta_{\sigma \downarrow} 2 I^{2} S \Lambda_{k \sigma}(\omega)}{1-I \Lambda_{k \sigma}(\omega)}\right)^{-1}$.

At the atomic limit $\left(\epsilon_{k}=0\right)$ and $\omega_{q} \rightarrow 0$ we obtain the two-pole solution given in ref. [6]:

$$
\begin{aligned}
G_{0}^{\text {at }} & =\frac{S+Z_{\sigma} S^{Z}+1}{2 S+1}(\omega+I S)^{-1} \\
& +\frac{S-Z_{\sigma} S^{Z}}{2 S+1}[\omega-I(S+1)]^{-1} .
\end{aligned}
$$

Moreover, at the first time in our theory we are able to calculate explicitly the full self-energy operator $\Sigma_{\sigma}(k, \omega)$ for the magnetic polaron problem (in the low-concentration limit)

$$
\sum_{\sigma}(k, \omega)=\frac{l^{2}}{N} \frac{M_{k \sigma}(\omega)}{1-\psi_{k \sigma}^{-1}(\omega) M_{k \sigma}(\omega)} .
$$

For brevity we write down only the $s-f$ exchange inelastic scattering contribution

$$
\begin{aligned}
& M_{k \sigma}^{s-\mathrm{f}}(\omega)=2\left(I^{2} S^{Z} / N\right) \\
& \quad \times \sum_{q p} \frac{\delta_{\sigma \downarrow}+\nu\left(\omega_{q}\right)}{\left[1-I \Lambda_{k \sigma}(\omega)\right]^{2}\left(\omega+Z_{\sigma} \omega_{q}-\epsilon_{k+q,-\sigma}\right)^{2}} \\
& \quad \times \frac{\left\langle S_{p}^{\sigma} S_{-p}^{-\sigma}\right\rangle}{\omega+Z_{\sigma}\left(\omega_{q}-\omega_{p}\right)-\epsilon_{k+q-p, \sigma}}
\end{aligned}
$$

where the longitudinal spin correlations are dropped for simplicity. A more detailed analysis will be published elsewhere.

\section{References}

[1] W. Nolting, Phys. Stat. Sol. 96b (1979) 11.

[2] S. Methfessel and D.C. Mattis, Handbuch der Physik $18 / 1$ (1968) 389.

[3] Yu.A. Izyumov and M.V. Medvedev, Sov. Phys. JETP 32 (1971) 302.

[4] P. Richmond, J. Phys. C3 (1970) 2402.

[5] B. Sriram Shastry and D.C. Mattis, Phys. Rev. B24 (1981) 5340.

[6] M.I. Auslender, M.I. Katsnelson and V.Uu. Irkhin, Physica 119B (1983) 309.

[7] N.M. Plakida, Phys. Lett. 43A (1973) 481.

[8] A.L. Kuzemsky, Theor. Math. Phys. 36 (1978) 208.

[9] D.N. Zubarev, Sov. Phys. Usp. 3 (1960) 320. 